\title{
Archéopages
}

Archéopages

Archéologie et société

\section{La restitution 3D et l'étude architecturale des bâtiments à parois rejetées en région Centre aux $\mathrm{II}^{\mathrm{e}}-\mathrm{I}^{\mathrm{er}} \mathrm{S}$. avant notre ère}

\section{Gaëlle Robert et Nicolas Holzem}

\section{(2) OpenEdition}

1 Journals

Édition électronique

URL : https://journals.openedition.org/archeopages/318

DOI : 10.4000/archeopages. 318

ISSN : 2269-9872

Éditeur

INRAP - Institut national de recherches archéologiques préventives

Édition imprimée

Date de publication : 1 mai 2013

Pagination : 89-93

ISSN : $1622-8545$

\section{Référence électronique}

Gaëlle Robert et Nicolas Holzem, « La restitution 3D et l'étude architecturale des bâtiments à parois

rejetées en région Centre aux $\| \mathrm{e}_{-1}{ }^{\mathrm{e}}$ s $\mathrm{L}$. avant notre ère », Archéopages [En ligne], 35 | 10/2012, mis en ligne le 01 octobre 2014, consulté le 22 janvier 2022. URL : http://journals.openedition.org/ archeopages/318; DOI : https://doi.org/10.4000/archeopages.318 


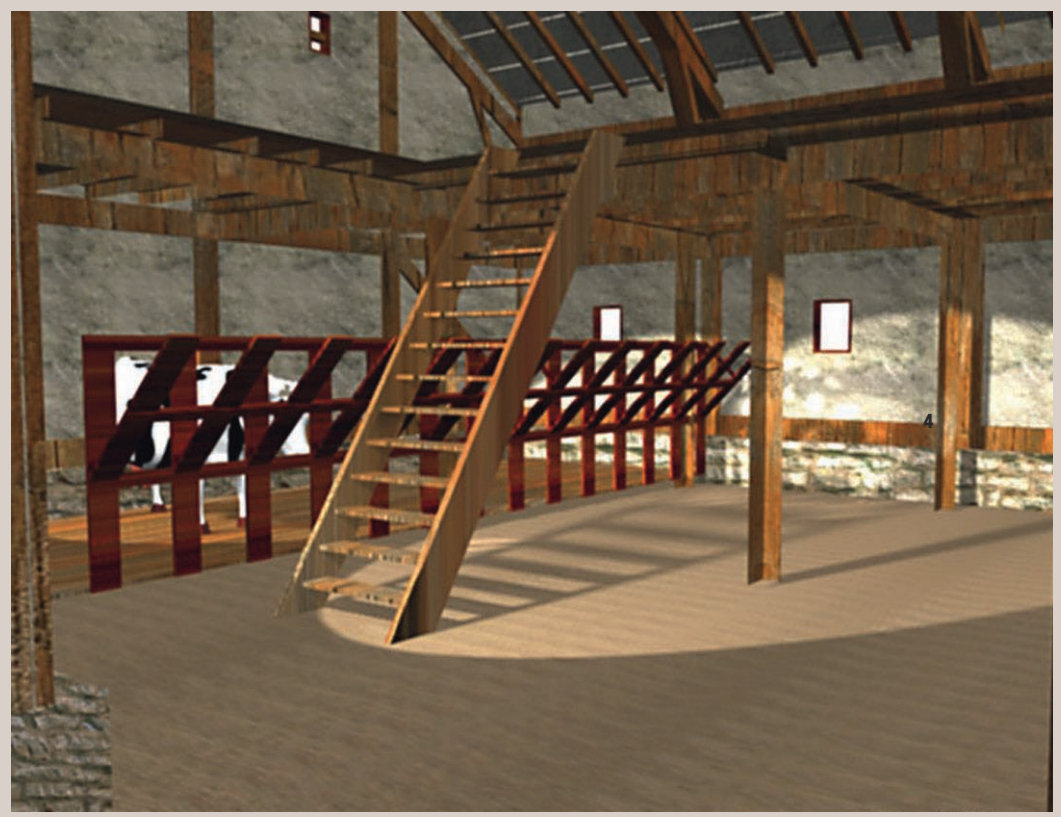

3.Proposition de restitution de l'étable.Cette image permet d'évoquer l'aspect probable de la partie réservée à la stabulation des bovidés. Séparée du reste de la grange par un râtelier, elle serait surmontée par un grenier en mezzanine destiné au stockage du fourrage.

a été dicté par le respect du module de base, en accord avec les proportions préconisées par les auteurs antiques. La restitution de la charpente de la grange repose sur l'implantation des poteaux axiaux et sur le module qui devait rythmer le pan de bois des façades. La découverte de tuiles dans les niveaux d'abandon a motivé le choix de la couverture. Enfin, la reconstitution des extensions périphériques reprend le système constitutif de la grange. Pour l'ensemble du bâtiment, l'implantation et la dimension des ouvertures relèvent à la fois des observations de fouille, de la logique climatique locale et des indications fournies par les traités d'agriculture antiques.

Cet exercice de restitution $3 \mathrm{D}$ a été présenté en septembre 2004 dans le cadre de la table ronde d'Insming (Moselle) organisée par l'ADRAL

(Association de recherche et de développement de l'archéologie en Lorraine) sur le thème «Restitution des bâtiments ruraux lorrains d'après les traces archéologiques : certitudes et incertitudes» (inédit). Certaines réactions de rejet ont permis d'appréhender combien il est important d'insister sur le fait que cette démarche n'est pas destinée à imposer une vision unique d'une réalité restituée. L'exercice soulève de nombreuses questions et oblige à proposer des réponses concrètes. Cependant, le résultat se doit d'être considéré comme la mise en image d'une hypothèse archéologique poussée à son terme, la création d'un support destiné à la réflexion et à l'ouverture des débats.

Cette première expérience positive s'est avérée riche en enseignements et a fait évoluer notre façon d'appréhender les fouilles suivantes. Ainsi, en 20082009, à l'occasion de la fouille des bains de la villa de Damblain (Vosges), la réflexion sur les restitutions des élévations a été ébauchée dès la phase de travail sur le terrain. Elle a permis d'attirer l'attention sur de nombreux indices, parfois ténus, et de porter les efforts de recherche sur certains matériaux parfois négligés, à l'exemple des enduits peints. Dès la phase de post-fouille, un travail commun de recherche mené avec les différents spécialistes a permis d'esquisser des propositions de restitution des élévations. Ce travail préparatoire a conduit naturellement à la mise en place d'un projet de restitution ${ }_{3} \mathrm{D}$ animée, actuellement réalisé en collaboration avec le SRA lorraine, l'Inrap et le CRAI (laboratoire de l'École nationale supérieure d'architecture de Nancy). Au terme de ce projet, l'outil de réflexion archéologique rejoindra la cause de la valorisation et de la communication auprès des spécialistes et du grand public.

La restitution $3 \mathrm{D}$ et l'étude architecturale des bâtiments à parois rejetées en région Centre aux $\mathrm{II}^{\mathrm{e}}-\mathrm{I}^{\mathrm{er}} \mathrm{S}$. avant notre ère

Gaëlle Robert, Inrap, UMR 7324 « Cités, Territoires, Environnement et Sociétés »

Nicolas Holzem, Inrap

La multiplication des fouilles en région Centre depuis une dizaine d'années sur la période du second âge du Fer a permis la découverte de très nombreux bâtiments datés des $\mathrm{II}^{\mathrm{e}} \mathrm{I}^{\mathrm{er}}$ siècles avant notre ère (Robert, 2009). Cette masse de données a conduit les archéologues spécialistes de la période à s'intéresser à l'architecture des bâtiments. La majorité de leurs plans, quadrangulaires avec quatre gros poteaux porteurs, semblent relativement homogènes. Ils présentent cependant des variations et un tableau typologique a pu être dressé à partir des découvertes du centre-ouest de la France pour cette période (Maguer, Robert, à paraître). Ainsi, les bâtiments d'habitation montrent le plus souvent un plan à quatre ou six poteaux de grandes dimensions, formant le module porteur de la construction. Des accès sont parfois marqués par une ou deux paires de poteaux moins profondément ancrés, disposés à l'extérieur du module porteur. Des traces de parois sont quelquefois observées, sous le forme de tranchées de fondation peu larges et peu profondes et/ou de trous de poteau. La surface réelle de ces bâtiments dépasse ainsi en général largement celle du module porteur, mais elle est souvent difficile à estimer.

Ce type de plan est désormais bien identifié dans la région. Toutefois, des recherches récemment publiées dans l'est de la France (Laurelut et al., 2005) ont permis d'observer une variation : le plan est toujours simple, carré ou rectangulaire à poteaux plantés, mais les supports du module porteur peuvent présenter une inclinaison légère, en moyenne de $10^{\circ}$ vers l'intérieur, comme sur le site de "Champ Chardon » ${ }^{\mathbf{1}}$ à Tours (Indre-et-Loire) [ill. 1]. Une dizaine de plans de ce type sont inventoriés dans le Centre, tous datés du $\mathrm{I}^{\mathrm{er}}$ siècle avant notre ère. Cette découverte fait désormais l'objet d'un intérêt particulier pour mieux comprendre les raisons de cette évolution du mode de construction.
1. Responsable scientifique : A. Couderc. 


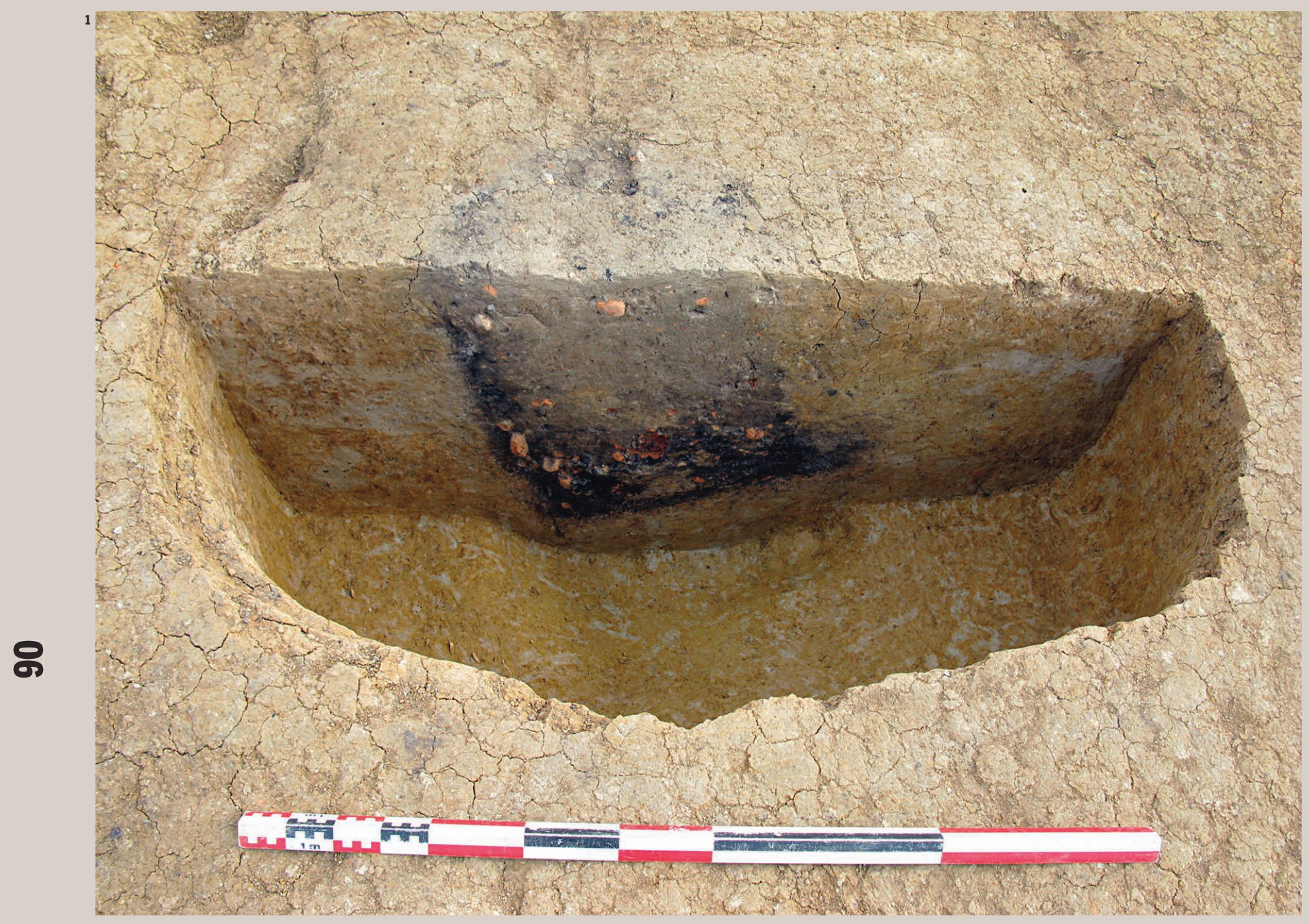

1. Plan et coupe d'un trou

de poteau dont le négatif

est incliné sur le site

de «Champ Chardon », Tours.

2. Exemple schématisé

de l'orientation différentielle

des axes de coupes d'un

bâtiment à module porteur

de quatre poteaux et double

accès avant sa fouille.

$\rightarrow$

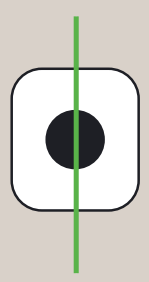

$\oplus$

$\oplus$

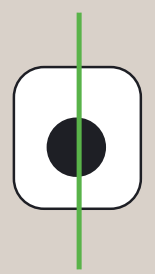

$\ominus$

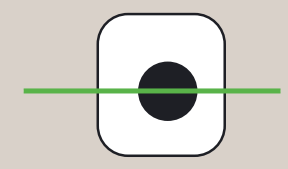




\section{L'utilité de l'étude architecturale}

La connaissance de l'architecture de l'âge du Fer dépend totalement des fouilles archéologiques. Les années 1970 et 1980 ont attiré l'attention sur ces questions (Audouze, Buchsenschutz, 1989) et de grands décapages extensifs ont mis au jour de nouvelles formes. Létude des trous de poteaux ne se limite pas à la reconnaissance des plans : en effet, les recherches portent désormais sur les modes de construction et leur évolution. La systématisation de la fouille des trous de poteau et de tout autre vestige se rapportant à l'architecture (tranchée, solin...) permet de poser les questions de la hauteur des parois, de la forme de la toiture, des types de matériaux, du statut de la construction, de sa durée d'occupation et éventuellement de sa fonction. Cette question reste largement dépendante de l'étude du mobilier.

Ces recherches sur l'habitat rural, développées principalement lors de grands travaux (ZAC, tracés linéaires), se font de manière inégale selon la zone d'étude : elles sont plus avancées sur le territoire carnute et turon que biturige. Toutefois, la fouille complète d'un enclos de La Tène finale à Déols (Indre) a permis en 2009 (Baguenier, 2011) d'appréhender l'architecture rurale biturige ; et celle d'Étrechet $^{2}$ (Indre), sur un site des $\mathrm{I}^{\mathrm{er}}$ et $\mathrm{II}^{\mathrm{e}}$ âges du Fer, a livré de grands bâtiments à module porteur de quatre poteaux avec des accès datés des $\mathrm{II}^{\mathrm{e}}-\mathrm{I}^{\mathrm{er}} \mathrm{S}$. avant notre ère (Cherdo, 2012). Certains possédaient encore des éléments de parois, matérialisées par des trous de poteau. Forte de l'expérience de Déols et d'autres fouilles en région Centre, l'équipe a abordé la problématique de l'architecture dès la fouille du site «Croc au Loup» à Étrechet.

\section{La mise en place d'un protocole de fouille}

La faisabilité d'une étude architecturale intégrant des hypothèses de restitution oblige à collecter en fouille toutes les données pour létude. L'accumulation d'expériences sur des sites de l'âge du Fer fouillés en région Centre a permis lélaboration d'un protocole de fouille des trous de poteau qui doit être la plus exhaustive possible. Les dimensions et la forme des creusements peuvent informer sur l'ancrage des poteaux au sol. Le négatif, s'il est perceptible, peut fournir les dimensions du poteau, sa forme, son inclinaison et donc sa fonction. La fouille fine permet d'appréhender les différentes phases: construction, occupation et abandon. Enfin le recueil du mobilier et l'observation de sa position permettent non seulement de préciser la datation, mais aussi de mieux comprendre l'architecture, en intégrant aux données mobilières les fragments de terre cuite, les gros charbons de bois, les clous, etc. La présence de poteaux porteurs massifs incite également à rechercher des trous de poteau plus ténus et des traces de paroi.

Dans ce but, les vestiges sont photographiés et relevés avant fouille et les axes de coupes des trous de poteau sont positionnés selon l'orientation du bâtiment [ill. 2] et de manière à percevoir d'éventuelles inclinaisons de poteau (Laurelut et al., 2007). La fouille, manuelle, doit différencier les strates de remplissage et permettre de ramasser le mobilier de manière exhaustive, y compris les restes de terre crue ou de charbon. Des photographies ou des relevés complémentaires peuvent être réalisés, en plus des traditionnels relevés et clichés de plan et coupe. L'ensemble des trous de poteau, si possible fouillés à $100 \%$, est systématiquement géoréférencé, y compris les plus douteux.

\section{L'étude des bâtiments après la fouille}

Les données recueillies sont intégralement utilisées dans l'étude architecturale. La description et linterprétation des bâtiments reposent entièrement sur ces éléments. Aussi, dans le cadre de la fouille d'Étrechet, un gain de temps considérable a été permis avec le $\mathrm{SIG}^{3}$, notamment grâce à la rapidité du montage des planches des bâtiments identifiés sur le site. Concernant la période des $\mathrm{II}^{\mathrm{e}}-\mathrm{I}^{\mathrm{er}}$ siècles avant notre ère, six bâtiments ont été repérés. Tous présentent des similarités avec leurs plans à module porteur et leurs poteaux massifs. Toutefois, chaque bâtiment possède ses caractéristiques propres permettant d'envisager des restitutions individuelles. C'est pourquoi tous les bâtiments sont décrits et interprétés en fonction des dimensions (surface du module porteur, valeur de la portée, profondeur conservée des trous de poteaux), mais aussi en considérant la stratigraphie et le mobilier. Cette première analyse a mis en évidence différentes questions (variations de la forme de la toiture et interaction entre les chevrons et le module porteur), auxquelles nous pouvons tenter de répondre via les hypothèses de restitution en $3 \mathrm{D}$. Les parois étant faiblement ancrées dans le sol, le module porteur concentre l'essentiel des poussées et implique donc des réponses techniques élaborées. Cette phase du travail, jusque-là menée par la personne qui étudiait les bâtiments, a été attribuée à un collègue dont les connaissances en SIG et en logiciel ${ }_{3} \mathrm{D}$ permettaient un gain de temps et un meilleur rendu des hypothèses?

\section{La restitution en ${ }_{3} D$}

Le recours à l'infographie $3 \mathrm{D}$ pouvant relever de trois objectifs différents, une réflexion en amont s'impose.

Premièrement, l'archivage des données par le relevé $3 \mathrm{D}$ de terrain ou par la numérisation d'objets au scanner sauvegardent des vestiges fragiles ou amenés à disparaître et permettent leur étude et/ou leur valorisation (tablettes d'argile, grottes ornées, etc.) ; il s'agit ici d'effectuer une copie numérique la plus fidèle possible du référent, ou bien de réaliser un simple relevé schématique tel le levé topographique.

Deuxièmement, la $3 \mathrm{D}$ permet la valorisation d'un site fouillé auprès du public par la reconstitution; cette version moderne de la maquette nécessite un rendu visuel travaillé, mais pas nécessairement détaillé: les détails seront rendus par des textures 


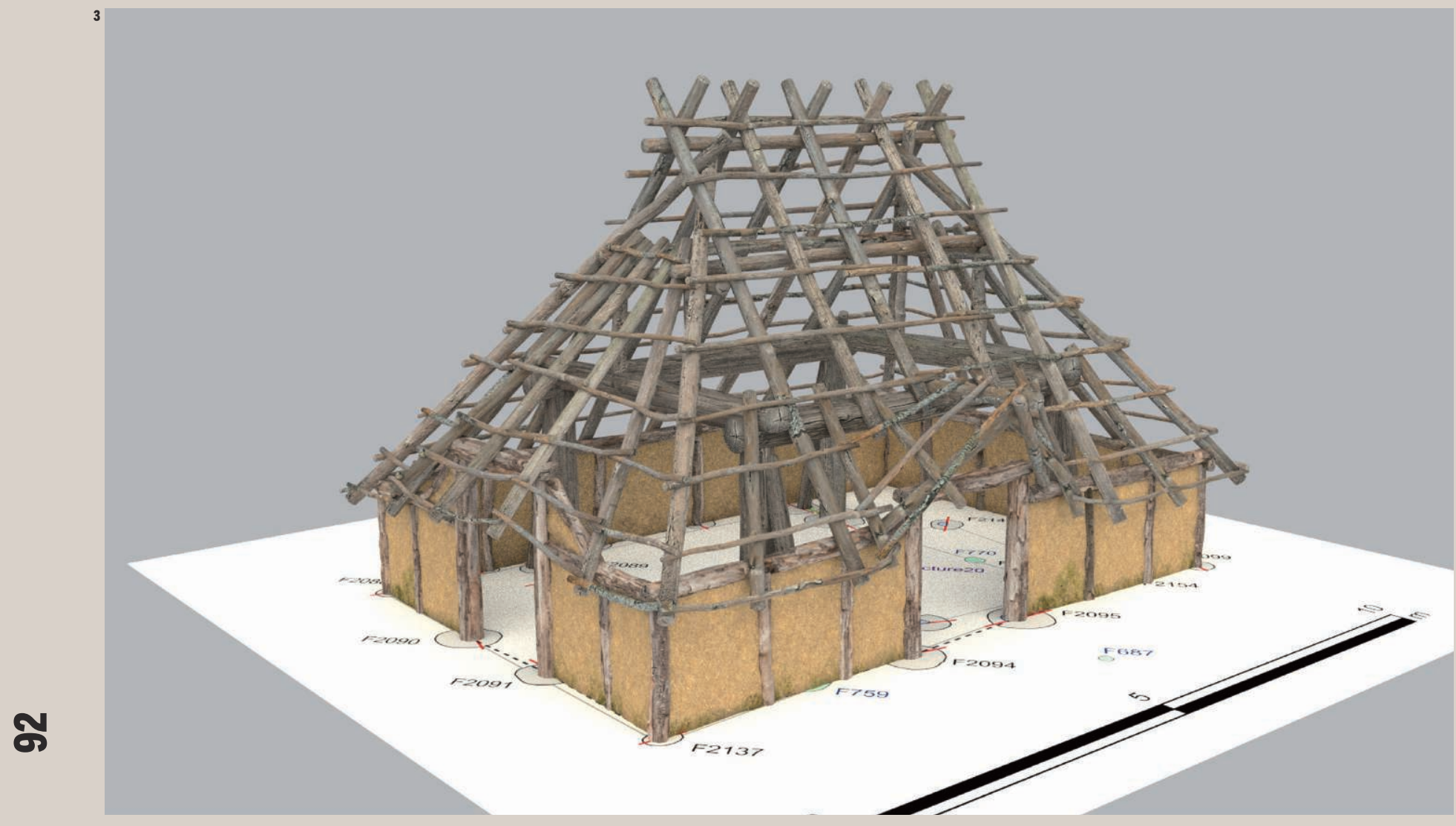

3. Hypothèse de restitution

en 3D de l'ensemble 16

du site d'Étrechet, basé sur le

plan du bâtiment (ci-dessous);

en blanc, la surface supposée

de son emprise au sol. Ici,

le module porteur présente

un assemblage de madriers

à mi-bois, ce quiévite l'usage

de clous (absents sur le site).

La toiture présente une

inclinaison à $60^{\circ}$, optimale

pour une couverture

de chaume.

(1)
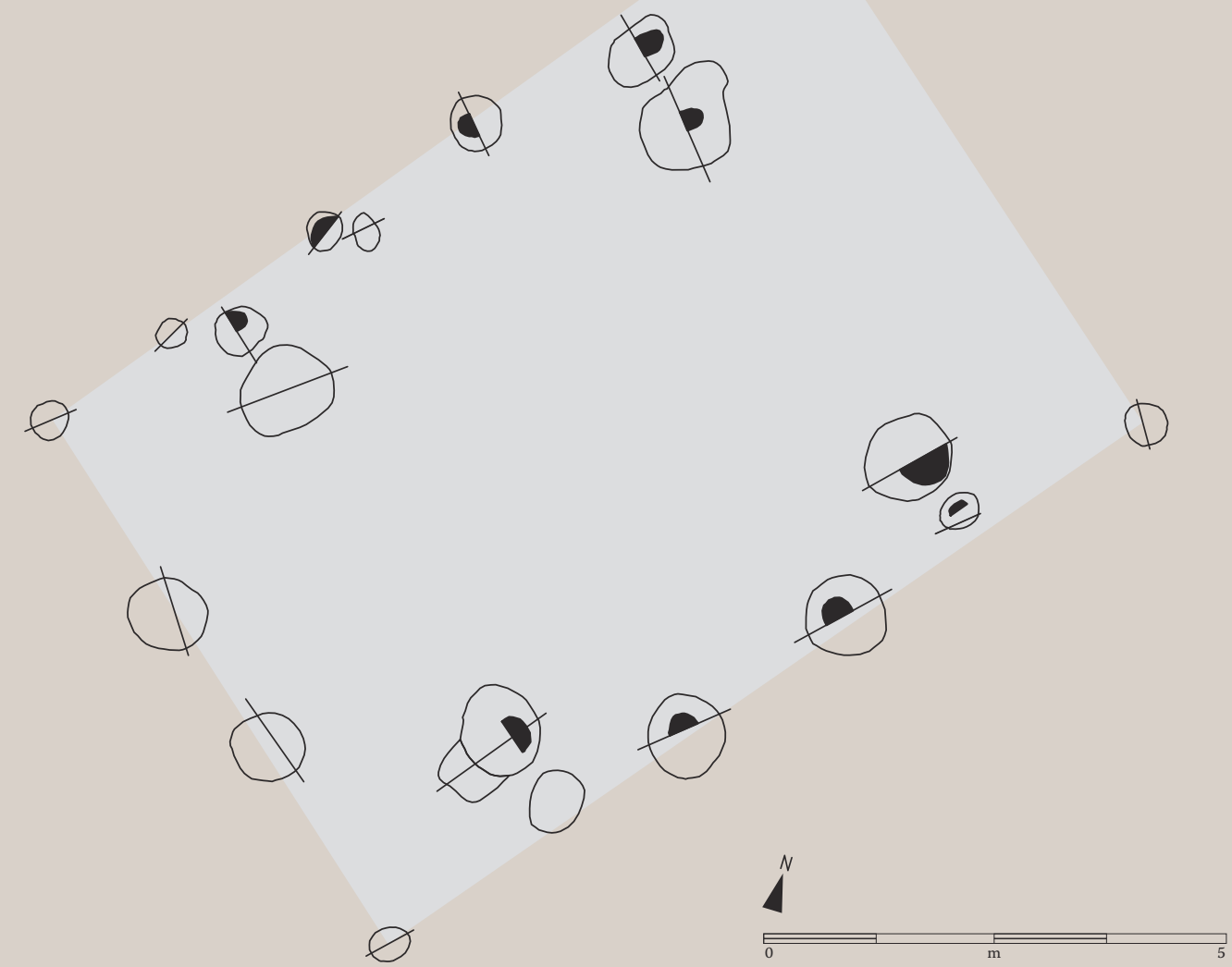
afin de diminuer les temps de calcul. Si ces restitutions se basent sur des données archéologiques, une large part est faite à l'imagination.

Enfin, la modélisation $3 \mathrm{D}$ peut être utilisée pour aider la recherche en testant des hypothèses de travail. L'infographiste est prioritairement contraint par la documentation archéologique qui peut être complétée par des sources iconographiques ou textuelles et par la comparaison à d'autres sites ou aux hypothèses de restitutions antérieures. Si le comparatisme ethnographique peut être utile (Épaud, 2009), il ne fait que compléter le champ des possibilités, techniques notamment.

Le niveau de détail pourra être très élevé à certains endroits et très faible à d'autres selon la documentation. Si le travail peut évidemment aboutir à des illustrations, ces dernières traduisent la réflexion menée en amont. Ainsi, dans la fouille d'Étrechet, les hypothèses de restitution des bâtiments sur poteaux concrétisaient le propos des archéologues, d'où le soin porté aux textures et un certain niveau de détail [ill. 3]. Pour le grand public, la couverture végétale des toitures aurait été restituée alors qu'elles ont ici été laissées nues afin de rendre visibles les propositions pour les charpentes.

La position des poteaux ancrés dans le sol ainsi que leurs diamètres ou leur éventuelle inclinaison proviennent des données de terrain. Les différentes hauteurs sont issues à la fois des observations directes, du dialogue avec le spécialiste ainsi que d'une logique architecturale (type de couverture, comme ici du chaume ou du roseau, ou mode d'assemblage de la structure). De même, nous sommes partis du principe qu'une personne de taille moyenne devait pouvoir se tenir debout partout. Ces contraintes se trouvent ainsi liées ; certaines sont fixes (la position des poteaux, par exemple), d'autres offrent une certaine variabilité aboutissant à une ou plusieurs propositions à la probabilité raisonnable.

Techniquement, la restitution des bâtiments d'Étrechet a été menée avec le logiciel libre Blender, aux possibilités très similaires à celles des logiciels propriétaires. La modélisation a été faite à échelle sur les plans directement importés dans le logiciel et les textures travaillées à partir de photographies. La $3 \mathrm{D}$ a permis de tester rapidement les hypothèses architecturales avant finalisation. La possibilité de déplacer une caméra selon l'angle de vue désiré et de rendre des vues orthographiques ou en perspective classique permet d'obtenir autant d'illustrations que nécessaire en un temps très court, ou bien encore des animations vidéo.

La restitution des bâtiments d'Étrechet s'est révélée très fructueuse pour un site où peu de données mobilières ont été recueillies. L'étude architecturale a permis de préciser les modes de construction sur le territoire biturige. Le travail a été orienté vers des hypothèses de restitutions en $3 \mathrm{D}$ pour mieux comprendre l'architecture sur poteaux plantés. La $3 \mathrm{D}$ a fait émerger différentes hypothèses portant notamment sur les formes des toitures et les assemblages. Elle a permis différents regards sur un type de plan apparemment simple, qui pose encore des questions d'interprétation. 